\title{
A Brunn-Minkowski Inequality for Symplectic Capacities of Convex Domains
}

\author{
Shiri Artstein-Avidan, Yaron Ostrover*
}

October 25, 2018

\begin{abstract}
In this work we prove a Brunn-Minkowski-type inequality in the context of symplectic geometry and discuss some of its applications.
\end{abstract}

\section{Introduction and Results}

In this note we examine the classical Brunn-Minkowski inequality in the context of symplectic geometry. Instead of considering volume, as in the original inequality, the quantity we are interested in is a symplectic capacity, given by the minimal symplectic area of a closed characteristic on the boundary of a convex domain. To explain the setting, the main results, and their significance, we start with an introduction.

\subsection{The Brunn-Minkowski inequality}

Denote by $\mathcal{K}^{n}$ the class of convex bodies in $\mathbb{R}^{n}$, that is, compact convex sets with non-empty interior. The Brunn-Minkowski inequality, in its classical formulation, states that if $K$ and $T$ are in $\mathcal{K}^{n}$, then

$$
(\operatorname{Vol}(K+T))^{\frac{1}{n}} \geq(\operatorname{Vol}(K))^{\frac{1}{n}}+(\operatorname{Vol}(T))^{\frac{1}{n}},
$$

where Vol denotes the $n$-dimensional volume (i.e, the Lebesgue measure) and the Minkowski sum of two bodies is defined by

$$
K+T=\{x+y: x \in K, y \in T\} .
$$

*The first and second named authors were both partially supported by BSF grant no. 2006079. This first named author was partially supported by the ISF grant No. 865/07, and the second named author was partially supported by NSF grant DMS-0706976. 
Moreover, equality holds if and only if $K$ and $T$ are homothetic, or in other words, coincide up to translation and dilation.

The Brunn-Minkowski inequality is a fundamental result in convex geometry and has innumerable applications, the most famous of which is probably a simple proof of the isoperimetric inequality. We recall that in fact it is known that the BrunnMinkowski inequality holds for any two measurable sets (while the equality condition requires convexity, or some special form of non-degeneracy). The inequality is connected with many other important inequalities such as the isoperimetric inequality, the Sobolev and the Log-Sobolev inequalities, and the Prékopa-Leindler inequality. Moreover, the Brunn-Minkowski inequality has diversified applications in analysis, geometry, probability theory, information theory, combinatorics, physics and more. We refer the reader to [15] for a detailed survey on this topic.

The importance of the Brunn-Minkowski inequality has led to efforts of finding analogous inequalities in other areas of mathematics, and recently, inequalities of Brunn-Minkowski type were proved for various well known functionals other than volume. Two examples of these functionals, which are related with calculus of variation and with elliptic partial differential equations, are the first eigenvalue of the Laplace operator [6] and the electrostatic capacity [5]. In this note we concentrate on a symplectic analogue of the inequality. To explain it, we turn now to the framework of symplectic geometry.

\subsection{Symplectic Capacities}

Consider the $2 n$-dimensional Euclidean space $\mathbb{R}^{2 n}$ with the standard linear coordinates $\left(x_{1}, y_{1}, \ldots, x_{n}, y_{n}\right)$. One equips this space with the standard symplectic structure $\omega_{s t}=\sum_{j=1}^{n} d x_{j} \wedge d y_{j}$, and with the standard inner product $g_{s t}=\langle\cdot, \cdot\rangle$. Note that under the identification between $\mathbb{R}^{2 n}$ and $\mathbb{C}^{n}$, these two structures are the real and the imaginary parts of the standard Hermitian inner product in $\mathbb{C}^{n}$, and $\omega(v, J v)=\langle v, v\rangle$, where $J$ is the standard complex structure on $\mathbb{R}^{2 n}$. Recall that a symplectomorphism of $\mathbb{R}^{2 n}$ is a diffeomorphism which preserves the symplectic structure i.e., $\psi \in \operatorname{Diff}\left(\mathbb{R}^{2 n}\right)$ such that $\psi^{*} \omega_{s t}=\omega_{s t}$. In what follows we denote by $\operatorname{Symp}\left(\mathbb{R}^{2 n}\right)$ the group of all the symplectomorphisms of $\mathbb{R}^{2 n}$.

Symplectic capacities are symplectic invariants which, roughly speaking, measure the symplectic size of subsets of $\mathbb{R}^{2 n}$. More precisely,

Definition 1.1. A symplectic capacity on $\left(\mathbb{R}^{2 n}, \omega_{\text {st }}\right)$ associates to each subset $U \subset \mathbb{R}^{2 n}$ a number $c(U) \in[0, \infty]$ such that the following three properties hold:

$(P 1) c(U) \leq c(V)$ for $U \subseteq V$ (monotonicity)

(P2) $c(\psi(U))=|\alpha| c(U)$ for $\psi \in \operatorname{Diff}\left(\mathbb{R}^{2 n}\right)$ such that $\psi^{*} \omega_{s t}=\alpha \omega_{s t}$ (conformality) 
(P3) $c\left(B^{2 n}(r)\right)=c\left(B^{2}(r) \times \mathbb{C}^{n-1}\right)=\pi r^{2}$ (nontriviality and normalization),

where $B^{2 k}(r)$ is the open $2 k$-dimensional Euclidean ball of radius $r$. Note that the third property disqualifies any volume-related invariant, while the first two properties imply that for two sets $U, V \subset \mathbb{R}^{2 n}$, a necessary condition for the existence of a symplectomorphism $\psi$ such that $\psi\left(U_{1}\right)=U_{2}$ is that $c\left(U_{1}\right)=c\left(U_{2}\right)$ for each symplectic capacity $c$.

A priori, it is unclear that symplectic capacities exist. The first example of a symplectic capacity is due to Gromov [16]. His celebrated non-squeezing theorem states that for $R>r$ the ball $B^{2 n}(R)$ does not admit a symplectic embedding into the symplectic cylinder $Z^{2 n}(r):=B^{2}(r) \times \mathbb{C}^{n-1}$. This theorem led to the following definitions:

Definition 1.2. The symplectic radius of a non-empty set $U \subset \mathbb{R}^{2 n}$ is

$$
c_{B}(U):=\sup \left\{\pi r^{2} \mid \text { There exists } \psi \in \operatorname{Symp}\left(\mathbb{R}^{2 n}\right) \text { with } \psi\left(B^{2 n}(r)\right) \subset U\right\} .
$$

The cylindrical capacity of $U$ is

$$
c^{Z}(U):=\inf \left\{\pi r^{2} \mid \text { There exists } \psi \in \operatorname{Symp}\left(\mathbb{R}^{2 n}\right) \text { with } \psi(U) \subset Z^{2 n}(r)\right\} .
$$

Note that both the symplectic radius and the cylindrical capacity satisfy the axioms of Definition 1.1 by the non-squeezing theorem. Moreover, it follows from Definition 1.1 that for every symplectic capacity $c$ and every open set $U \subset \mathbb{R}^{2 n}$ we have $c_{B}(U) \leq c(U) \leq c^{Z}(U)$.

The above axiomatic definition of symplectic capacities is originally due to Ekeland and Hofer [12]. Nowadays, a variety of symplectic capacities are known to exist. For several of the detailed discussions on symplectic capacities we refer the reader to [7], [17], [18], [20], [22] and [29].

In this note we mainly concentrate on two important examples of symplectic capacities which arose from the study of periodic solutions of Hamiltonian systems. These are the Ekeland-Hofer capacity $c_{E H}$ introduced in [12], [13] and the HoferZehnder capacity $c_{H Z}$ introduced in [19]. These invariants have several applications, among them a new proof of Gromov's non-squeezing theorem, establishing the existence of closed characteristics on or near an energy surface, and studying the Hofer geometry on the group of Hamiltonian diffeomorphisms (see e.g [18]). Moreover, it is known that on the class of convex bodies in $\mathbb{R}^{2 n}$, these two capacities coincide, and can be represented by the minimal symplectic area of a closed characteristic on the boundary of the convex domain. Since in this note we are concerned only with convex sets, we omit the general definitions of these two capacities, and give a definition which coincides with the standard ones on the class of convex domains. This is done in Theorem 1.3 below. Next we turn to some background on Hamiltonian dynamics. 


\subsection{Hamiltonian Dynamics on Convex Domains}

Let $U$ be a bounded, connected, open set in $\mathbb{R}^{2 n}$ with smooth boundary containing the origin. A nonnegative function $F: \mathbb{R}^{2 n} \rightarrow \mathbb{R}$ is said to be a defining function for $U$ if it satisfies that $\partial U=F^{-1}(1)$, that $U=F^{-1}([0,1])$, and that 1 is a regular value of $F$. Next, let $F$ be a defining function for $U$, and denote by $X_{F}=J \nabla F$ the corresponding Hamiltonian vector field defined by $i_{X_{F}} \omega=-d F$. Note that $X_{F}$ is always tangent to $\partial U$ since $d F(x) \cdot X_{F}(x)=-\omega\left(X_{F}(x), X_{F}(x)\right)=0$ for all $x \in \partial U$, and hence it defines a non-vanishing vector field on $\partial U$. It is well known (see e.g [18]) that the orbits of this vector field, that is, the solutions of the classical Hamiltonian equation $\dot{x}=X_{F}(x)$, do not depend, up to parametrization, on the choice of the Hamiltonian function $F$ representing $\partial U$. Indeed, if $H$ is another defining function for $\partial U$ i.e.,

$$
\partial U=\{x ; H(x)=1\}=\{x ; F(x)=1\} \text { with } d H, d F \neq 0 \text { on } \partial U
$$

and where 1 is a regular value of both $F$ and $H$, then $d F(x)=\lambda(x) d H(x)$ at every point $x \in \partial U$, with $\lambda(x) \neq 0$, and therefore $X_{F}=\lambda X_{H}$ on $\partial U$ where $\lambda \neq 0$. Thus, the two vector fields have, up to reparametrization, the same solutions on $\partial U$.

The images of the periodic solutions of the above mentioned Hamiltonian equation are called the "closed characteristics" of $\partial U$ (where periodic means $T$-periodic for some positive $T$ ). The breakthrough in the global existence of closed characteristics was achieved by Weinstein [30] and Rabinowitz [25] who established the existence of a closed characteristic on every convex (and in fact also on every star-shaped) hypersurface in $\mathbb{R}^{2 n}$.

We recall the following definition. The action of a $T$-periodic solution $l(t)$ is defined by (see e.g. [18] Page 7):

$$
\mathcal{A}(l)=\int_{l} \lambda=\frac{1}{2} \int_{0}^{T}\langle-J \dot{l}(t), l(t)\rangle d t,
$$

where $\lambda=\sum_{1}^{n} x_{i} d y_{i}$ is the Liouville 1 -form whose differential is $d \lambda=\omega$. This action of a periodic orbit $l(t)$ is the symplectic area of a disc spanned by the loop $l(t)$.

In particular, it is a symplectic invariant i.e., $\mathcal{A}(\psi(l))=\mathcal{A}(l)$, for any $\psi \in$ $\operatorname{Symp}\left(\mathbb{R}^{2 n}\right)$.

We next introduce the Ekeland-Hofer and the Hofer-Zehnder capacities, denoted by $c_{E H}$ and $c_{H Z}$ respectively. As stated above, instead of presenting the general definitions of these two capacities we present a definition sufficient for our purpose which coincides with the standard ones on the class of convex domains. This definition follows from the theorem below, which is a combination of results from [12] and [18]. 
Theorem 1.3. Let $K \subset \mathbb{R}^{2 n}$ be a convex bounded domain with smooth boundary $\partial K$. Then there exists at least one closed characteristic $\gamma^{*} \subset \partial K$ satisfying

$c_{E H}(K)=c_{H Z}(K)=\mathcal{A}\left(\gamma^{*}\right)=\min \{|\mathcal{A}(l)|: l$ is a closed characteristic on $\partial K\}$

Such a closed characteristic, which minimizes the action (note that there might be more than one), is called throughout this text a "capacity carrier" for $K$. In addition, we refer to the coinciding Ekeland-Hofer and Hofer-Zehnder capacities on the class of convex domains as the Ekeland-Hofer-Zehnder capacity, and denote it from here onwards, when there is no possibility for confusion with a general capacity, by $c$.

\subsection{Main Results}

A natural question following from the discussion above is whether a Brunn-Minkowski type inequality holds for the symplectic-size of sets, which is given by their symplectic capacities. In this paper we restrict ourselves to the class of convex domains and to the Ekeland-Hofer-Zehnder capacity. However, we do not exclude the possibility that the Brunn-Minkowski inequality holds for other symplectic capacities or other, more general classes of bodies in $\mathbb{R}^{2 n}$. For example, in dimension 2 , any symplectic capacity agrees with the volume for a large class of sets in $\mathbb{R}^{2}$ (see [27]), and hence the BrunnMinkowski inequality holds for this class. Also, it is not difficult to verify that for the linearized ball capacity (for a definition see [3], 2]) the Brunn-Minkowski inequality holds.

The main result in this paper is the following: Denote by $\mathcal{K}^{2 n}$ the class of compact convex bodies in $\mathbb{R}^{2 n}$ which has non-empty interior.

Theorem 1.4. Let $c$ be the Ekeland-Hofer-Zehnder capacity. Then for any $n$, and any $K, T \in \mathcal{K}^{2 n}$, one has

$$
c(K+T)^{\frac{1}{2}} \geq c(K)^{\frac{1}{2}}+c(T)^{\frac{1}{2}} .
$$

Moreover, equality holds if and only if $K$ and $T$ have a pair of homothetic capacity carriers.

In fact, Theorem 1.4 is a special case of a slightly more general result which we now describe. For a convex body $K$, denote by $\|x\|_{K}:=\inf \{r: x / r \in K\}$ the corresponding gauge function. Moreover, we uniquely associate with $K$ its support function $h_{K}$ given by:

$$
h_{K}(u)=\sup \{\langle x, u\rangle: x \in K\}, \text { for all } u \in \mathbb{R}^{2 n} .
$$

Note that this is no other than the gauge function of the polar body

$$
K^{\circ}=\left\{x \in \mathbb{R}^{2 n}:\langle x, y\rangle \leq 1, \text { for every } y \in K\right\},
$$


or, in the symmetric case, simply the dual norm $h_{K}(u)=\|u\|_{K}^{*}=\|u\|_{K^{\circ}}$.

In [14], Firey introduced a new operation for convex bodies, called " $p$-sum", which depends on a parameter $p \geq 1$ and extends the classical Minkowski sum. For two convex bodies $K, T \in \mathbb{R}^{2 n}$, both containing the origin, the $p$-sum of $K$ and $T$, denoted $K+{ }_{p} T$, is defined via its support function in the following way:

$$
h_{K+{ }_{p} T}(u)=\left(h_{K}^{p}(u)+h_{T}^{p}(u)\right)^{\frac{1}{p}}, \quad u \in \mathbb{R}^{2 n} .
$$

The convexity of $h_{K+{ }_{p} T}$ follows easily from Minkowski's inequality. The case $p=1$ corresponds to the classical Minkowski sum. Thus, Theorem 1.4 is a special case of the following:

Theorem 1.5. Let $c$ be the Ekeland-Hofer-Zehnder capacity. Then for any $n$, any $p \geq 1$, and any $K, T \in \mathcal{K}^{2 n}$ one has

$$
c\left(K+_{p} T\right)^{\frac{p}{2}} \geq c(K)^{\frac{p}{2}}+c(T)^{\frac{p}{2}} .
$$

Moreover, equality holds if and only if $K$ and $T$ have a pair of homothetic capacity carriers.

An interesting corollary of Theorem 1.4 is a symplectic analogue of the classical isoperimetric inequality comparing volume and surface area which we now present. For a curve $\gamma:[0, T] \rightarrow \mathbb{R}^{2 n}$ and a convex body $K$ including 0 in its interior we denote by length ${ }_{K}(\gamma)=\int_{0}^{T}\|\dot{\gamma}(t)\|_{K} d t$ the length of $\gamma$ with respect to the body $K$. The following corollary is proven in Section 4 .

Corollary 1.6. For any $K, T \in \mathcal{K}^{2 n}$, and $c$ as above,

$$
4 c(K) c(T) \leq\left(\text { length }_{J T^{\circ}}(\gamma)\right)^{2},
$$

where $\gamma$ is any capacity carrier of $K$.

In Section 4 we explain why Corollary 1.6 can be thought of as a consequence of an isoperimetric-type inequality for capacities. Note that in the special case where $T$ is the Euclidean unit ball, Equation (1.4.5) becomes

$$
4 \pi c(K) \leq(\operatorname{length}(\gamma))^{2}
$$

where $\gamma$ is any capacity carrier for $K$ and where length stands for the standard Euclidean length. This last consequence is known, and can be deduced from the standard isoperimetric inequality in $\mathbb{R}^{2 n}$ combined with the well known fact that the symplectic area is always less than or equal to the Euclidean area. 
Another special case of Corollary 1.6 which can be useful is the following: let $K$ be a symplectic ellipsoid $E=\sum_{i=1}^{n} \frac{x_{i}^{2}+y_{i}^{2}}{r_{i}^{2}}$, where $1=r_{1} \leq r_{2}, \ldots \leq r_{n}$. Equation (1.4.5 implies that for any $T \in \mathcal{K}^{2 n}$

$$
4 \pi c(T) \leq \operatorname{length}_{J T^{\circ}}\left(S^{1}\right)^{2}
$$

where $S^{1}$ is the capacity carrier of $E$ given by $x_{1}^{2}+y_{1}^{2}=1$. Moreover, since the same is true for any symplectic image of $E$, we get that

$$
4 \pi c(T) \leq \inf _{\varphi \in \operatorname{Symp}\left(\mathbb{R}^{2 n}\right)}\left(\text { length }_{J T^{\circ}}\left(\varphi\left(S^{1}\right)\right)\right)^{2} .
$$

This estimate is sometimes strictly better than other available estimates, such as volume radius (see [28, [2], 3]).

Next we state another corollary of Theorem 1.4, which improves a result previously proved in [3] by other methods. Define the "mean-width" of a centrally symmetric convex body $K$ to be

$$
M^{*}(K):=\int_{S^{2 n-1}} \max _{y \in K}\langle x, y\rangle \sigma(d x)
$$

where $\sigma$ is the rotationally invariant probability measure on the unit sphere $S^{2 n-1}$. In other words, we integrate over all unit directions $x$ half of the distance between two parallel hyperplanes touching $K$ and perpendicular to the vector $x$. Mean-width is an important parameter in Asymptotic Geometric Analysis, and is the geometric version of a central probabilistic parameter, see e.g. [24]. We show in Section 4 below that:

Corollary 1.7. For every centrally symmetric convex body $K \subset \mathcal{K}^{2 n}$, one has

$$
c(K) \leq \pi\left(M^{*}(K)\right)
$$

Moreover, equality holds if and only if $K$ is a Euclidean ball.

In fact, as the proof will demonstrate, this corollary follows from standard arguments once we have a Brunn-Minkowski-type inequality. The same is true for the following result.

Corollary 1.8. For any two symmetric convex bodies $K, T \subset \mathcal{K}^{2 n}$, one has for every $x \in \mathbb{R}^{2 n}$ that

$$
c(K \cap(x+T)) \leq c(K \cap T) .
$$

More generally, for any $K, T \in \operatorname{cal} K^{2 n}$ any $x, y \in \mathbb{R}^{2 n}$ and any $0 \leq \lambda \leq 1$, we have that

$$
\lambda c^{1 / 2}(K \cap(x+T))+(1-\lambda) c^{1 / 2}(K \cap(y+T)) \leq c^{1 / 2}(K \cap(\lambda x+(1-\lambda) y+T)) .
$$


We wish to remark that this note can be considered as a continuation of the line of work which was presented in [2] and [3], in which we used methods and intuition coming from the field of asymptotic geometric analysis to obtain results in symplectic geometry.

Structure of the paper: The paper is organized as follows. In Section 2 we introduce the main ingredient in the proof of our main theorem. In Section 3 we prove the Brunn-Minkowski inequality for the Ekeland-Hofer-Zehnder capacity $c$ and characterize the equality case. In Section 4 we prove the above mentioned applications of the inequality, and in the last section we prove a technical lemma.

Acknowledgment: We cordially thank Leonid Polterovich for very helpful remarks.

\section{The Main Ingredient}

In this section we introduce the main ingredient in the proof of Theorem 1.5,

We note that there is no loss of generality in assuming, from here onwards, that in addition to being compact and with non-empty interior, all convex bodies considered also have a smooth boundary and contain the origin in the interior. Indeed, affine translations in $\mathbb{R}^{2 n}$ are symplectomorphisms, which accounts for the assumption that the origin is in the interior. Secondly, once we know the Brunn-Minkowski inequality for smooth convex domains, the general case follows by standard approximation, since symplectic capacities are continuous on the class of convex bodies with respect to the Hausdorff distance (see e.g. [23], Page 376).

The main ingredient in the proof of Theorem 1.5, is the following proposition which is another characterization of the Ekeland-Hofer-Zehnder capacity, valid for smooth convex sets. Let $W^{1, p}\left(S^{1}, \mathbb{R}^{2 n}\right)$ be the Banach space of absolutely continuous $2 \pi$-periodic functions whose derivatives belong to $L_{p}\left(S^{1}, \mathbb{R}^{2 n}\right)$.

Proposition 2.1. Let $c$ be the Ekeland-Hofer-Zehnder capacity. For any convex body $K \subset \mathbb{R}^{2 n}$ with smooth boundary, and any two parameters $p_{1}>1, p_{2} \geq 1$

$$
c(K)^{\frac{p_{2}}{2}}=\pi^{p_{2}} \min _{z \in \mathcal{E}_{p_{1}}} \frac{1}{2 \pi} \int_{0}^{2 \pi} h_{K}^{p_{2}}(\dot{z}(t)) d t
$$

where

$$
\mathcal{E}_{p_{1}}=\left\{z \in W^{1, p_{1}}\left(S^{1}, \mathbb{R}^{2 n}\right): \int_{0}^{2 \pi} z(t) d t=0, \frac{1}{2} \int_{0}^{2 \pi}\langle J z(t), \dot{z}(t)\rangle d t=1\right\} .
$$

In the case where $p_{1}=p_{2}=2$, a proof of the above proposition can be found in [18] and [21]. There the authors use the idea of dual action principle by Clarke [8] 
in order to prove the existence of a closed characteristic for convex surfaces, a claim originally due to Rabinowitz [25] and Weinstein [30]. For further discussions on Clarke's dual action principle, and in particular its use for the proof of existence of closed characteristics, see e.g. [11] and the references within.

It turns out that the special case $p_{1}=p_{2}>1$ implies the more general case of possibly different $p_{1}>1, p_{2} \geq 1$. That is, we claim that the following Proposition formally implies Proposition 2.1:

Proposition 2.2. For any convex body $K \subset \mathbb{R}^{2 n}$ with smooth boundary, and $p>1$

$$
c(K)^{\frac{p}{2}}=\pi^{p} \min _{z \in \mathcal{E}_{p}} \frac{1}{2 \pi} \int_{0}^{2 \pi} h_{K}^{p}(\dot{z}(t)) d t .
$$

Proof of the implication Proposition 2.2 $\Rightarrow$ Proposition 2.1. Note that for $1<p_{2} \leq p_{1}$, one has $\mathcal{E}_{p_{1}} \subset \mathcal{E}_{p_{2}}$. Moreover, from Hölder's inequality it follows that

$$
\left(\frac{1}{2 \pi} \int_{0}^{2 \pi} h_{K}^{p_{2}}(\dot{z}(t)) d t\right)^{\frac{1}{p_{2}}} \leq\left(\frac{1}{2 \pi} \int_{0}^{2 \pi} h_{K}^{p_{1}}(\dot{z}(t)) d t\right)^{\frac{1}{p_{1}}} .
$$

Therefore, from Proposition 2.2 it follows that for $1<p_{2} \leq p_{1}$

$$
\begin{aligned}
c(K)^{\frac{1}{2}} & =\pi \min _{z \in \mathcal{E}_{p_{1}}}\left(\frac{1}{2 \pi} \int_{0}^{2 \pi} h_{K}^{p_{1}}(\dot{z}(t)) d t\right)^{\frac{1}{p_{1}}} \\
& \geq \pi \min _{z \in \mathcal{E}_{p_{1}}}\left(\frac{1}{2 \pi} \int_{0}^{2 \pi} h_{K}^{p_{2}}(\dot{z}(t)) d t\right)^{\frac{1}{p_{2}}} \\
& \geq \pi \min _{z \in \mathcal{E}_{p_{2}}}\left(\frac{1}{2 \pi} \int_{0}^{2 \pi} h_{K}^{p_{2}}(\dot{z}(t)) d t\right)^{\frac{1}{p_{2}}}=c(K)^{\frac{1}{2}} .
\end{aligned}
$$

In particular, we have equality throughout. Similarly,

$$
\begin{aligned}
c(K)^{\frac{1}{2}} & =\pi \min _{z \in \mathcal{E}_{p_{1}}}\left(\frac{1}{2 \pi} \int_{0}^{2 \pi} h_{K}^{p_{1}}(\dot{z}(t)) d t\right)^{\frac{1}{p_{1}}} \\
& \geq \pi \min _{z \in \mathcal{E}_{p_{2}}}\left(\frac{1}{2 \pi} \int_{0}^{2 \pi} h_{K}^{p_{1}}(\dot{z}(t)) d t\right)^{\frac{1}{p_{1}}} \\
& \geq \pi \min _{z \in \mathcal{E}_{p_{2}}}\left(\frac{1}{2 \pi} \int_{0}^{2 \pi} h_{K}^{p_{2}}(\dot{z}(t)) d t\right)^{\frac{1}{p_{2}}}=c(K)^{\frac{1}{2}} .
\end{aligned}
$$

Thus, we conclude that for any $1<p_{1}, p_{2}$

$$
c(K)^{\frac{1}{2}}=\pi \min _{z \in \mathcal{E}_{p_{1}}}\left(\frac{1}{2 \pi} \int_{0}^{2 \pi} h_{K}^{p_{2}}(\dot{z}(t)) d t\right)^{\frac{1}{p_{2}}} .
$$

To complete the proof we need only to explain the case of $p_{2}=1$. On the one hand, Hölder's inequality implies that

$$
c(K)^{\frac{1}{2}}=\pi \min _{z \in \mathcal{E}_{p_{1}}}\left(\frac{1}{2 \pi} \int_{0}^{2 \pi} h_{K}^{p_{1}}(\dot{z}(t)) d t\right)^{\frac{1}{p_{1}}} \geq \pi \min _{z \in \mathcal{E}_{p_{1}}} \frac{1}{2 \pi} \int_{0}^{2 \pi} h_{K}(\dot{z}(t)) d t .
$$


On the other hand, using that $\lim (\min ) \leq \min (\lim )$, we can let $1<p_{2} \rightarrow 1$ in equation (2.3 . By Lebesgue's dominated convergence theorem we can also insert the limit into the integral and get that

$$
c(K)^{\frac{1}{2}} \leq \pi \min _{z \in \mathcal{E}_{p_{1}}} \frac{1}{2 \pi} \int_{0}^{2 \pi} h_{K}(\dot{z}(t)) d t,
$$

which completes the proof of Proposition 2.1 in the case of $p_{2}=1$.

Before turning to the proof of Proposition 2.2, which will be our main objective throughout the rest of this section, let us point out an important consequence of the above argument which will be helpful for us later (especially in the proof of the equality case for the Brunn-Minkowski inequality).

Fix $p_{1}>1$ and let $\tilde{z}$ be any path in $\mathcal{E}_{p_{1}}$ for which the minimum is attained in equation (2.3). Letting $1 \leq p_{2}<p_{1}$ we get that

$$
\begin{aligned}
c(K)^{\frac{1}{2}} & =\pi\left(\frac{1}{2 \pi} \int_{0}^{2 \pi} h_{K}^{p_{1}}(\dot{\tilde{z}}(t)) d t\right)^{\frac{1}{p_{1}}} \\
& \geq \pi\left(\frac{1}{2 \pi} \int_{0}^{2 \pi} h_{K}^{p_{2}}(\dot{\tilde{z}}(t)) d t\right)^{\frac{1}{p_{2}}} \\
& \geq \pi \min _{z \in \mathcal{E}_{p_{1}}}\left(\frac{1}{2 \pi} \int_{0}^{2 \pi} h_{K}^{p_{2}}(\dot{z}(t)) d t\right)^{\frac{1}{p_{2}}}=c(K)^{\frac{1}{2}} .
\end{aligned}
$$

In particular, there is equality in the first inequality so that the $L_{p_{1}}$ and $L_{p_{2}}$ norms of the function $h_{K}(\dot{\tilde{z}}(t))$ coincide. This clearly implies that this function is constant in $t$. Another fact which easily follows from the line of inequalities above is that the minimum is attained on the same paths $z$ for all $p_{2} \geq 1$ (in particular, on a function which belongs to $\bigcap_{p>1} \mathcal{E}_{p}$ ).

Thus, we have shown that Proposition 2.2 implies the following

Corollary 2.3. Fix $p_{1}>1$ and $p_{2} \geq 1$. Any path $\tilde{z}$ which minimizes $\int_{0}^{2 \pi} h_{K}^{p_{2}}(\dot{z}(t)) d t$ over $\mathcal{E}_{p_{1}}$ satisfies that the function $h_{K}(\dot{z}(t))$ is the constant function $c(K) / \pi$, and in particular all the $L_{p}$ norms of the function $h_{K}(\dot{z}(t))$ coincide.

After the proof of Proposition 2.2, we will give a geometrical explanation to this fact, see Remark 2.7 below.

We now turn to the proof of Proposition 2.2. We follow closely the arguments, valid for $p=2$, in [18] and [21]. Fix $p>1$ and consider the functional

$$
I_{p}(z)=\int_{0}^{2 \pi} h_{K}^{p}(\dot{z}(t)) d t
$$

defined on the space $\mathcal{E}_{p}$, which was defined in the statement of Proposition 2.1. A key ingredient in the proof is Lemma 2.5 below, which we will prove in Section 5 , 
and which gives a one-to-one correspondence between the so called "critical points" of the functional $I_{p}$ and closed characteristics on $\partial K$. Before stating the lemma, we must define what we mean by a critical point of $I_{p}$, since $\mathcal{E}_{p}$ is not closed under all perturbations.

Definition 2.4. An element $z \in \mathcal{E}_{p}$ is called a critical point of $I_{p}$ if the following holds: For every $\xi \in W^{1, p}\left(S^{1}, \mathbb{R}^{2 n}\right)$ satisfying $\int_{0}^{2 \pi} \xi(t) d t=0$ and $\int_{0}^{2 \pi}\langle\xi(t), J \dot{z}(t)\rangle d t=0$, one has

$$
\int_{0}^{2 \pi}\left\langle\nabla h_{K}^{p}(\dot{z}(t)), \dot{\xi}(t)\right\rangle=0
$$

To understand why this definition is natural, first notice that the above condition $\int_{0}^{2 \pi}\langle\xi(t), J \dot{z}(t)\rangle d t=0$ implies that $\int\langle\dot{\xi}(t), J z(t)\rangle d t=0$ by integration by parts. Next, consider the element $z_{\varepsilon}=z+\varepsilon \xi$. It belongs to $W^{1, p}\left(S^{1}, \mathbb{R}^{2 n}\right)$ and satisfies the normalization condition $\int_{0}^{2 \pi} z_{\varepsilon}(t) d t=0$, but its action is not normalized to be 1 , thus it is not necessarily in $\mathcal{E}_{p}$. However, its action is close to 1 with difference being of order $o(\varepsilon)$. Indeed,

$$
\left|\mathcal{A}\left(z_{\varepsilon}\right)\right|=\frac{1}{2} \int_{0}^{2 \pi}\left\langle J z_{\varepsilon}(t), \dot{z}_{\varepsilon}(t)\right\rangle d t=1+\frac{\varepsilon^{2}}{2} \int_{0}^{2 \pi}\langle J \xi(t), \dot{\xi}(t)\rangle d t
$$

Denote by $z_{\varepsilon}^{\prime}$ the normalized path:

$$
z_{\varepsilon}^{\prime}=\frac{z_{\varepsilon}}{1+\frac{\varepsilon^{2}}{2} \int\langle J \xi(t), \dot{\xi}(t)\rangle d t}
$$

Note that now $z_{\varepsilon}^{\prime} \in \mathcal{E}_{p}$. For a critical point, it is natural to require that the difference between $I_{p}(z)$ and $I_{p}\left(z_{\varepsilon}^{\prime}\right)$ will be of order $o(\varepsilon)$. Taking the first order approximation we have

$$
\left.I_{p}\left(z_{\varepsilon}^{\prime}\right)=\int_{0}^{2 \pi} h_{K}^{p}\left(\dot{z}_{\varepsilon}^{\prime}\right)(t)\right) d t=\int_{0}^{2 \pi} h_{K}^{p}(\dot{z}(t)) d t+\varepsilon \int_{0}^{2 \pi}\left\langle\nabla h_{K}^{p}(\dot{z}(t)), \dot{\xi}(t)\right\rangle+o\left(\varepsilon^{2}\right),
$$

and for the second term on the right hand side to disappear we need exactly the condition in the definition of a critical point above. In particular, we emphasize that the minimum of $I_{p}$ over $\mathcal{E}_{p}$ is attained at a critical point according to our definition, a fact which will be important in the proof. With the definition in hand, we may formulate the following lemma, which for $p=2$ appears in [18], Pages 26-30. For the sake of completion we include its proof for general $p>1$ in Section 5 .

Lemma 2.5. Let $K \subset \mathbb{R}^{2 n}$ be a convex body with smooth boundary, and fix $p>1$. Each critical point of the functional $I_{p}(z)$ satisfies the Euler equation

$$
\nabla h_{K}^{p}(\dot{z})=\frac{p}{2} \lambda J z+\alpha, \quad \text { where } \quad \lambda=I_{p}(z),
$$


for some fixed vector $\alpha$ (which may be different for different critical points), and vice versa: each point z satisfying Equation 2.4 is a critical point of $I_{p}$. Moreover, the functional $I_{p}(z)$ achieves its minimum i.e., there is $\tilde{z} \in \mathcal{E}_{p}$ such that

$$
I_{p}(\tilde{z})=\inf _{z \in \mathcal{E}_{p}} I(z)=\int_{0}^{2 \pi} h_{K}^{p}(\dot{\tilde{z}}(t)) d t=\tilde{\lambda} \neq 0,
$$

and in particular, $\tilde{z}$ satisfies Equation 2.4.

Proof of Proposition 2.2 The idea is as follows: we define an invertible mapping $\mathcal{F}$ between critical points $z$ of $I_{p}(z)$ and closed characteristics $l$ on the boundary of $K$. Moreover, we will show that the action of $l=\mathcal{F}(z)$ is a simple monotone increasing function of $I_{p}(z)$. In particular, the critical point $z$ for which the minimum of $I_{p}(z)$ is attained is mapped to the closed characteristic minimizing the action. Since the minimal action of a closed characteristic is exactly the Ekeland-Hofer-Zehnder capacity, the result will follow.

To define the mapping $\mathcal{F}$, let $z: S^{1} \rightarrow R^{2 n}$ be a critical point of $I_{p}$. In particular from Lemma 2.5 we have that

$$
\nabla h_{K}^{p}(\dot{z})=\frac{p}{2} \lambda J z+\alpha,
$$

for some vector $\alpha$ and $\lambda=I_{p}(z)$. We will use the Legendre transform in order to define an affine linear image of $z$ which is a closed characteristic on the boundary $\partial K$ of $K$, which we will then define as $\mathcal{F}(z)$. Recall that the Legendre transform is defined as follows: For $f: \mathbb{R}^{n} \rightarrow \mathbb{R}$, one defines

$$
\mathcal{L} f(y)=\sup _{x \in \mathbb{R}^{n}}[\langle y, x\rangle-f(x)], \forall y \in \mathbb{R}^{n} .
$$

It is not hard to check that

$$
\left(\mathcal{L}\left(h_{K}^{p}\right)\right)(v)=\frac{p^{1-q}}{q} h_{K^{\circ}}^{q}(v),
$$

where $p^{-1}+q^{-1}=1$ and $K^{\circ}$ is, as before, the polar body of $K$. Note that $h_{K^{\circ}}^{q}$ is a defining function of $K$ (that is, $K$ is its 1-level set) which is homogeneous of degree $q$. After applying the Legendre transform and using the fact that $v=\nabla h_{K}^{p}(u)$ is inverted point-wise by $u=\nabla \mathcal{L} h_{K}^{p}(v)$ equation (2.4) becomes:

$$
\dot{z}=\frac{p^{1-q}}{q} \nabla h_{K^{\circ}}^{q}\left(\frac{p}{2} \lambda J \tilde{z}+\alpha\right)=\nabla h_{K^{\circ}}\left(\frac{q^{\frac{1}{1-q}}}{2} \lambda J z+\frac{\alpha q^{\frac{1}{1-q}}}{p}\right) .
$$

Next, let

$$
l=\kappa\left(\frac{q^{\frac{1}{1-q}}}{2} \lambda J z+\frac{\alpha q^{\frac{1}{1-q}}}{p}\right),
$$


where $\kappa$ is a positive normalization constant which we will readily choose. Differentiating (2.6) we see that $l$ satisfies the following Hamiltonian equation.

$$
\dot{l}=\frac{\kappa}{2} q^{\frac{1}{1-q}} \lambda J \nabla h_{K^{\circ}}^{q}(l / \kappa)=\frac{\kappa^{2-q}}{2} q^{\frac{1}{1-q}} \lambda J \nabla h_{K^{\circ}}^{q}(l) .
$$

Note that $l$ is a periodic trajectory of the Hamiltonian equation corresponding to the Hamiltonian function $h_{K^{\circ}}^{q}$. Since we ask $l \in \partial K$ we need to choose $\kappa$ such that $l$ will lie in the energy level $h_{K^{\circ}}^{q}=1$. For this purpose, note that since $h_{K^{\circ}}^{q}$ is homogeneous of degree $q$ we obtain from Euler's formula that

$$
\begin{aligned}
\frac{1}{2 \pi} \int_{0}^{2 \pi} h_{K^{\circ}}^{q}(l(t)) d t & =\frac{1}{2 \pi q} \int_{0}^{2 \pi}\left\langle\nabla h_{K^{\circ}}^{q}(l(t)), l(t)\right\rangle d t=-\frac{\kappa^{q-2} q^{\frac{1}{q-1}}}{\pi \lambda q} \int_{0}^{2 \pi}\langle j \dot{J}(t), l(t)\rangle d t \\
& =\frac{q^{\frac{1}{1-q}} \kappa^{q}}{4 \pi q \lambda} \int_{0}^{2 \pi}\left\langle\lambda \dot{z}(t), \lambda J z(t)+\frac{2 \alpha}{p}\right\rangle d t=\frac{\kappa^{q} \lambda q^{\frac{q}{1-q}}}{2 \pi}
\end{aligned}
$$

which is equal to 1 if we choose $\kappa=(2 \pi / \lambda)^{\frac{1}{q}} q^{\frac{1}{q-1}}$. Therefore, for this value of $\kappa$ we have that

$$
l=\left(\frac{2 \pi}{\lambda}\right)^{\frac{1}{q}}\left(\frac{\lambda}{2} J z+\frac{\alpha}{p}\right)
$$

is a closed trajectory of the Hamiltonian equation corresponding to the Hamiltonian $h_{K^{\circ}}^{q}$ on $\partial K$. This $l$ we denote by $\mathcal{F}(z)$. (To be completely formal, to agree with the way closed characteristics were defined, we let $\mathcal{F}(z)$ be the image of $l$ in $\mathbb{R}^{2 n}$.) Below we will show that this mapping is invertible, and compute $\mathcal{F}^{-1}$.

Next we derive the relation between $\mathcal{A}(l)$ and $\lambda=I_{p}(z)$. Using Euler's formula again, and the above value of $\kappa$ we conclude that

$$
\mathcal{A}(l)=\frac{1}{2} \int_{0}^{2 \pi}\langle-J \dot{l}(t), l(t)\rangle d t=\frac{\kappa^{2}}{8} q^{\frac{2}{1-q}} \lambda^{2} \int_{0}^{2 \pi}\left\langle\dot{z}(t), J z(t)+\frac{2 \alpha}{\lambda p}\right\rangle d t=4^{-\frac{1}{p}}(\pi)^{\frac{2}{q}} \lambda^{\frac{2}{p}}
$$

Equivalently,

$$
\mathcal{A}^{\frac{p}{2}}(l)=1 / 2(\pi)^{\frac{p}{q}} \lambda
$$

In order to show that the map $\mathcal{F}$ (we should actually write $\mathcal{F}_{p}$ as it depends on $p$, but we omit this index so as not to overload notation) is indeed one-to-one and onto, we now define $\mathcal{F}^{-1}$. Starting now with a closed characteristic $\Gamma$ on $\partial K$, it is not difficult to check that we may assume using a standard re-parametrization argument that it is the image of a loop $l$ with $l:[0,2 \pi] \rightarrow \mathbb{R}^{2 n}$ and $i=d J \nabla h_{K^{\circ}}^{q}(l)$, for some constant $d$. Next, we define

$$
\mathcal{F}^{-1}(l)=J^{-1}\left((\pi d q)^{-1 / 2}\left(l-\frac{1}{2 \pi} \int_{0}^{2 \pi} l(t) d t\right)\right) .
$$

We will show that this map is mapping closed characteristics to critical points. Set $z=\mathcal{F}^{-1}(l)$. It is easy to check that $\int_{0}^{2 \pi} z(t) d t=0$. The fact that $z \in W^{1, p}\left(S^{1}, \mathbb{R}^{2 n}\right)$ 
follows from the boundedness of $l$ (as Image $(l) \in \partial K$ is bounded) and the following argument: since $\dot{z}=C_{1} \cdot \nabla h_{K^{\circ}}^{q}(l)$ for some constant $C_{1}$ and, $\nabla h_{K^{\circ}}^{q}$, being homogenous of degree $q-1$, satisfy $\left|\nabla h_{K^{\circ}}^{q}(x)\right| \leq C_{2}|x|^{q-1}$ for some constant $C_{2}$, we conclude that for some constants $C_{3}$ and $C_{4}$,

$$
\int_{0}^{2 \pi}|\dot{z}(t)|^{p} d t=C_{3} \int_{0}^{2 \pi}\left|\nabla h_{K^{\circ}}^{q}(l(t))\right|^{\frac{q}{q-1}} d t \leq C_{4} \int_{0}^{2 \pi}|l(t)|^{q} d t<\infty .
$$

Moreover

$$
\begin{aligned}
\mathcal{A}(z) & =\frac{1}{2} \int_{0}^{2 \pi}\langle\dot{z}(t), J z(t)\rangle d t=\frac{1}{2}(\pi d q)^{-1} \int_{0}^{2 \pi}\left\langle J^{-1} i(t), l(t)\right\rangle d t \\
& =\frac{1}{2}(\pi d q)^{-1} \int_{0}^{2 \pi}\left\langle d \nabla h_{K^{\circ}}^{q}(l(t)), l(t)\right\rangle d t=\frac{1}{2 \pi} \int_{0}^{2 \pi} h_{K^{\circ}}^{q}(l(t))=1,
\end{aligned}
$$

where the next to last inequality follows from Euler's formula. Finally, note that

$$
\dot{z}=(\pi d q)^{-\frac{1}{2}} J^{-1} \dot{l}=(\pi d q)^{-\frac{1}{2}} d \nabla h_{K^{\circ}}^{q}(l)=(\pi d q)^{-\frac{1}{2}} d \nabla h_{K^{\circ}}^{q}\left((\pi d q)^{\frac{1}{2}} J z+\int_{0}^{2 \pi} l(t) d t\right)
$$

Using the Legendre transform as before we get that

$$
\nabla h_{K}^{p}(\dot{z})=\alpha J z+\beta,
$$

where $\alpha$ and $\beta$ are constants (depending on $d$ and $q$ ). Moreover,

$$
I_{p}(z)=\int_{0}^{2 \pi} h_{K}^{p}(\dot{z}(t)) d t=\frac{1}{p} \int_{0}^{2 \pi}\left\langle\nabla h_{K}^{p}(\dot{z}(t)), \dot{z}(t)\right\rangle d t=\frac{\alpha}{p} \int_{0}^{2 \pi}\langle J z(t), \dot{z}(t)\rangle d t=\frac{2 \alpha}{p}
$$

From Lemma 2.5 it now follows that $z$ is a critical point of $I_{p}$ and hence the map $\mathcal{F}^{-1}$ is well defined. It is not difficult to show that for every critical point, $\mathcal{F}^{-1} \mathcal{F}(z)=z$ and that for every closed characteristic, $\mathcal{F F}^{-1}(l)=l$, and we omit this computation.

This one-to-one correspondence, and the monotone relation between $\mathcal{A}(\mathcal{F}(z))$ and $I_{p}(z)$, implies that the for $\tilde{z}$, a critical point for which the minimum of $I(z)$ is attained, its "dual" $\tilde{l}=\mathcal{F}(\tilde{z})$ has minimal action among all closed characteristics $l$. This fact together with Theorem 1.3 (which we consider, for the purpose of this note, as the definition of Ekeland-Hofer-Zehnder capacity of convex domains) implies that

$$
c(K)^{\frac{p}{2}}=\mathcal{A}^{\frac{p}{2}}(\tilde{l})=(1 / 2)^{p}(2 \pi)^{\frac{p}{q}} \lambda=\pi^{p} \frac{1}{2 \pi} \int_{0}^{2 \pi} h_{K}^{p}(\dot{\tilde{z}}(t)) d t .
$$

The proof of the proposition is now complete.

Remark 2.6. In the proof we have shown the following fact, which we will use later in the note once again: For every $p$, there exists an invertible mapping $\mathcal{F}\left(=\mathcal{F}_{p}\right)$, mapping critical points of $I$ (which by Lemma 2.5 are exactly the loops satisfying equation (2.4)), to closed characteristics on $\partial K$, and moreover, satisfying

$$
\mathcal{A}^{\frac{p}{2}}(\mathcal{F}(z))=(1 / 2)^{p}(2 \pi)^{\frac{p}{q}} I_{p}(z) .
$$


Remark 2.7. The fact exhibited in Corollary 2.3, which might seem surprising at first, can be geometrically explained (for the case $p>1$ ) after the above construction has been made. Indeed, recall that (fixing some $p>1$ ) for every path $z \in \mathcal{E}_{p}$ which is a critical point of $I_{p}$ there corresponds a path $l \in \partial K, l=\mathcal{F}_{p}(z)$, which is a linear image of $z$. Moreover, by the above formulae, putting all the constants together and naming them $A_{1}, A_{2}, A_{3}$, we have (using that $h_{K^{\circ}}(l)=1$ )

$$
\dot{z}=A_{1} J^{-1} \dot{l}=A_{2} \nabla h_{K^{\circ}}^{q}(l)=A_{3} \nabla h_{K^{\circ}}(l) .
$$

Thus, we have $h_{K}(\dot{z})=A_{3} h_{K}\left(\nabla h_{K^{\circ}}(l)\right)$. However, a simple fact from Convexity (See [26], Corollary 1.7.3. Page 40) is that for a convex body $T$ and a vector $0 \neq u \in \mathbb{R}^{n}$, the gradient of the dual norm in a certain direction is exactly the support of the body in this direction. More formally:

$$
\nabla h_{T}(u)=\left\{x \in T: h_{T}(u)=\langle x, u\rangle\right\}
$$

which in our case, by smoothness, is simply one point (on the boundary of $T$, of course). Thus, in particular, $h_{K}\left(\nabla h_{K^{\circ}}(u)\right)=h_{K^{\circ}}(u)$, and we see that

$$
h_{K}(\dot{z})=A_{3} h_{K^{\circ}}(l)=A_{3},
$$

is constant and does not depend on $t$, as claimed in Corollary 2.3 and proven there by other means.

\section{Proof of the Main result}

In this section we use Proposition 2.1 to prove our main theorem.

Proof of Theorem 1.5. Fix $p_{1}>1$. It follows from equation (1.4.3) that for every $z \in \mathcal{E}_{p_{1}}$ and $p \geq 1$

$$
\frac{1}{2 \pi} \int_{0}^{2 \pi} h_{K{ }_{p} T}^{p}(\dot{z}(t)) d t=\frac{1}{2 \pi} \int_{0}^{2 \pi} h_{K}^{p}(\dot{z}(t)) d t+\frac{1}{2 \pi} \int_{0}^{2 \pi} h_{T}^{p}(\dot{z}(t)) d t
$$

By multiplying both sides of the above equation by $\pi^{p}$, taking the minimum over all $z \in \mathcal{E}_{p_{1}}$, and applying Proposition 2.1 above, we conclude that for every $p \geq 1$

$$
c\left(K+{ }_{p} T\right)^{\frac{p}{2}} \geq c(K)^{\frac{p}{2}}+c(T)^{\frac{p}{2}} .
$$

In particular, for $p=1$ we get the Brunn-Minkowski inequality for the Ekeland-HoferZehnder capacity.

We turn now to prove the equality case. We start by proving that if $K$ and $T$ have homothetic capacity carriers, then equality holds in $(3.2)$ for every $p \geq 1$. 
Let $\Gamma_{K} \subset \partial K$ be a capacity carrier for $K$. As in the proof of Proposition 2.2 above, we can choose a parameterized curve representing $\Gamma_{K}$ via $\Gamma_{K}=$ Image $l_{K}$, where $i_{K}=d_{K} J \nabla h_{K^{\circ}}^{2}\left(l_{K}\right)$ and $l_{K}(0)=l_{K}(2 \pi)$. Moreover, it follows from the proof of Proposition 2.2 (say in the case $p=2$ ) that for every such $l_{K}$ there is corresponding minimizer $z_{k} \in \mathcal{E}_{2}$ of the functional $I_{2}$ :

$$
z_{K}:=\mathcal{F}^{-1}\left(l_{K}\right)=J^{-1}\left(\left(2 \pi d_{K}\right)^{-1 / 2}\left(l_{K}-\frac{1}{2 \pi} \int_{0}^{2 \pi} l_{K}(t) d t\right)\right),
$$

such that

$$
c(K)^{\frac{1}{2}}=\pi \min _{z \in \mathcal{E}_{2}}\left(\frac{1}{2 \pi} \int_{0}^{2 \pi} h_{K}^{2}(\dot{z}(t)) d t\right)^{\frac{1}{2}}=\pi\left(\frac{1}{2 \pi} \int_{0}^{2 \pi} h_{K}^{2}\left(\dot{z}_{K}(t)\right) d t\right)^{\frac{1}{2}}
$$

Moreover, combining this with Proposition 2.1 and Corollary 2.3 we conclude that for every $p \geq 1$ :

$$
c(K)^{\frac{p}{2}}=\pi^{p} \min _{z \in \mathcal{E}_{2}} \frac{1}{2 \pi} \int_{0}^{2 \pi} h_{K}^{p}(\dot{z}(t)) d t=\pi^{p} \frac{1}{2 \pi} \int_{0}^{2 \pi} h_{K}^{p}\left(\dot{z}_{K}(t)\right) d t .
$$

Similarly, let $\Gamma_{T}$ be a capacity carrier for $T$, set $l_{T}$ the corresponding parameterized curve which represents $\Gamma_{T}$, and let $z_{T}=\mathcal{F}^{-1}\left(l_{T}\right) \in \mathcal{E}_{2}$ be the corresponding critical point of $I_{2}$ which satisfies

$$
c(T)^{\frac{p}{2}}=\pi^{p} \min _{z \in \mathcal{E}_{2}} \frac{1}{2 \pi} \int_{0}^{2 \pi} h_{T}^{p}(\dot{z}(t)) d t=\pi^{p} \frac{1}{2 \pi} \int_{0}^{2 \pi} h_{T}^{p}\left(\dot{z}_{T}(t)\right) d t .
$$

Note that in order to have equality in (3.2), it is enough to show that $z_{K}=z_{T}$. To this end we observe that since $\Gamma_{K}$ and $\Gamma_{T}$ are homothetic, there exist two constants $\alpha$ and $\beta$ such that $l_{T}=\alpha l_{K}+\beta$. This implies that $z_{T}=\left(\frac{d_{K}}{d_{T}}\right)^{1 / 2} \alpha z_{K}$ and that $\mathcal{A}\left(l_{T}\right)=\alpha^{2} \mathcal{A}\left(l_{K}\right)$. Moreover, since $\Gamma_{K}$ and $\Gamma_{T}$ are capacity carriers of $K$ and $T$ respectively, it follows that $\mathcal{A}\left(l_{K}\right)=c(K)$ and $\mathcal{A}\left(l_{T}\right)=c(T)$. Hence, we conclude that $\alpha=\left(\frac{c(T)}{c(K)}\right)^{1 / 2}$. On the other hand

$$
\begin{aligned}
c(K) & =\mathcal{A}\left(l_{K}\right)=\frac{1}{2 \pi} \int_{0}^{2 \pi}\left\langle l_{K}(t), J l_{K}(t)\right\rangle d t=\frac{1}{2 \pi} \int_{0}^{2 \pi}\left\langle l_{K}(t), d_{K} \nabla h_{K^{\circ}}^{2}\left(l_{K}(t)\right)\right\rangle d t \\
& =\frac{2 d_{K}}{2 \pi} \int_{0}^{2 \pi} h_{K^{\circ}}^{2}\left(l_{K}(t)\right) d t=2 d_{K},
\end{aligned}
$$

and similarly $c(T)=2 d_{T}$. This implies that $z_{K}=z_{T}$ and hence we have an equality in 3.2 for every $p \geq 1$ as required.

Next, we assume that equality holds in (3.2) for some $p \geq 1$ and prove that $K$ and $T$ have homothetic capacity carriers.

Let $p_{1}>1$ and $p \geq 1$. Note that equality in (3.2) implies that

$$
\min _{z \in \mathcal{E}_{p_{1}}} \int_{0}^{2 \pi} h_{K{ }_{p} T}^{p}(\dot{z}(t)) d t=\min _{z \in \mathcal{E}_{p_{1}}} \int_{0}^{2 \pi} h_{K}^{p}(\dot{z}(t)) d t+\min _{z \in \mathcal{E}_{p_{1}}} \int_{0}^{2 \pi} h_{T}^{p}(\dot{z}(t)) d t
$$


This in turn implies that there exists $\tilde{z} \in \mathcal{E}_{p_{1}}$ such that

$$
\min _{z \in \mathcal{E}_{p_{1}}} \int_{0}^{2 \pi} h_{K}^{p}(\dot{z}(t)) d t=\int_{0}^{2 \pi} h_{K}^{p}(\dot{\tilde{z}}(t)) d t \text { and } \min _{z \in \mathcal{E}_{p_{1}}} \int_{0}^{2 \pi} h_{T}^{p}(\dot{z}(t)) d t=\int_{0}^{2 \pi} h_{T}^{p}(\dot{\tilde{z}}(t)) d t
$$

Combining this fact with Proposition 2.1, we conclude that

$$
\min _{z \in \mathcal{E}_{p_{1}}}\left(\int_{0}^{2 \pi} h_{K}^{p_{1}}(\dot{z}(t)) d t\right)^{\frac{1}{p_{1}}}=\min _{z \in \mathcal{E}_{p_{1}}}\left(\int_{0}^{2 \pi} h_{K}^{p}(\dot{z}(t)) d t\right)^{\frac{1}{p}}=\left(\int_{0}^{2 \pi} h_{K}^{p}(\dot{\tilde{z}}(t))\right)^{\frac{1}{p}}
$$

In other words, $\tilde{z} \in \mathcal{E}_{p_{1}}$ is a critical point of the functional $I_{p_{1}}^{K}=\int_{0}^{2 \pi} h_{K}^{p_{1}}(\dot{z}(t)) d t$ defined on the space $\mathcal{E}_{p_{1}}$, where $p_{1}>1$. It follows from the proof of Proposition [2.2 together with (2.8) and (2.9), that for such $\tilde{z}$ their is a corresponding $\tilde{l}_{K}$ which satisfies

$$
\tilde{l}_{K}=\left(\frac{2 \pi}{\lambda_{K}}\right)^{\frac{1}{q_{1}}}\left(\frac{\lambda}{2} J \tilde{z}+\frac{\alpha_{K}}{p_{1}}\right)=c(K)^{\frac{1}{2}} J \tilde{z}+A_{K}
$$

where $A_{K}$ is a constant which depends on $K, p_{1}$, and $q_{1}^{-1}+p_{1}^{-1}=1$. Similarly, since $\tilde{z}$ is also a critical point of $I_{p_{1}}^{T}$, we have that $\tilde{l}_{T}=c(T)^{\frac{1}{2}} J \tilde{z}+A_{T}$. We conclude that $\tilde{l}_{T}=\alpha \tilde{l}_{K}+\beta$ where $\alpha=c(T)^{\frac{1}{2}} / c(K)^{\frac{1}{2}}$. This implies that $K$ and $T$ have homothetic capacity carriers and the proof of Theorem 1.5 is now complete.

\section{Corollaries of the Main Theorem}

In this section we prove Corollaries [1.6, 1.7 and 1.8. We start with the proof of Corollary 1.6. As in the case of the classical isoperimetric inequality, which connects the surface area of a body and its volume, the Brunn-Minkowski inequality is useful in obtaining a lower bound for the derivative of the volume-type function. This follows directly from Theorem 1.4 above and the following computation: for any convex bodies $K$ and $T$ in $\mathbb{R}^{2 n}$ and any $\varepsilon>0$,

$$
\frac{c(K+\varepsilon T)^{\frac{1}{2}}-c(K)^{\frac{1}{2}}}{\varepsilon} \geq \frac{c(K)^{\frac{1}{2}}+\varepsilon c(T)^{\frac{1}{2}}-c(K)^{\frac{1}{2}}}{\varepsilon}=c(T)^{\frac{1}{2}} .
$$

The limit on the left hand side as $\varepsilon \rightarrow 0^{+}$can be thought of as a "directional derivative" of $c^{1 / 2}$ in the "direction" $T$. Note that this argument holds for any symplectic capacity for which one is able to show that the Brunn-Minkowski inequality holds. However, to get a meaningful result, one must find a geometric interpretation for the so-called derivative which one arrives at. To be more precise, let us define, for a convex body $T$, the functional $d_{T}(K)$ by

$$
d_{T}(K)=\lim _{\varepsilon \rightarrow 0^{+}} \frac{c(K+\varepsilon T)-c(K)}{\varepsilon} .
$$

Inequality (4.1) implies the following easy corollary: 
Corollary 4.1. For every convex body $K \subset \mathbb{R}^{2 n}$, one has

$$
d_{T}(K)=\left.2 c(K)^{\frac{1}{2}} \frac{d}{d \varepsilon} c(K+\varepsilon T)^{\frac{1}{2}}\right|_{\varepsilon=0^{+}} \geq 2 c(K)^{\frac{1}{2}} c(T)^{\frac{1}{2}}
$$

The only part which requires justification is the existence of the limit. Let us show that $c^{1 / 2}(K+\varepsilon T)$ has derivative at $\varepsilon=0^{+}$(which is, since $c(K) \neq 0$, the same as showing that $c(K+\varepsilon T)$ has a derivative): Let $s<t$, note that

$$
\frac{c(K+t T)^{\frac{1}{2}}-c(K)^{\frac{1}{2}}}{t} \leq \frac{c(K+s T)^{\frac{1}{2}}-c(K)^{\frac{1}{2}}}{s},
$$

is equivalent to

$$
(s / t)\left(c(K+t T)^{\frac{1}{2}}\right)+(1-s / t) c(K)^{\frac{1}{2}} \leq c(K+s T)^{\frac{1}{2}},
$$

which follows from the Brunn-Minkowski inequality. Hence, the expression in the limit is a decreasing function of $\varepsilon>0$, and converges to its supremum as $\varepsilon \rightarrow 0^{+}$, provided it is bounded. Showing that it is bounded is simple, since $T \subset R K$ for some $R>0$ (which can be huge, and may depend on the dimension) and thus

$$
\frac{c(K+t T)^{\frac{1}{2}}-c(K)^{\frac{1}{2}}}{t} \leq \frac{c(K+t R K)^{\frac{1}{2}}-c(K)^{\frac{1}{2}}}{t}=R .
$$

This completes the proof of Corollary 4.1.

One way to use Corollary 4.1 is to find a geometric interpretation of the derivative of the capacity, $d$. Roughly speaking, if $c$ is a symplectic "volume", $d$ should be a kind of symplectic "surface-area". Instead, what we do below is to bound $d$ from above by an expression with a clear geometric meaning: minimal length of loops in a certain norm (as in the statement of Corollary 1.6), and then Corollary 4.1 gives a lower bound, in terms of capacity, of this expression.

We fix $\varepsilon>0, p_{1}>1$ and $p_{2}=1$, and denote by $\tilde{z} \in \mathcal{E}_{p_{1}}$ any path on which the minimum in Equation (2.1] is attained for $c(K)^{\frac{1}{2}}$. We compute:

$$
\begin{aligned}
c(K+\varepsilon T)^{\frac{1}{2}} & =\pi \min _{z \in \mathcal{E}_{p_{1}}} \frac{1}{2 \pi} \int_{0}^{2 \pi} h_{K+\varepsilon T}(\dot{z}(t)) d t \\
& =\pi \min _{z \in \mathcal{E}_{p_{1}}} \frac{1}{2 \pi} \int_{0}^{2 \pi} h_{K}(\dot{z}(t)) d t+\varepsilon h_{T}(\dot{z}(t)) d t \\
& \leq \pi \frac{1}{2 \pi} \int_{0}^{2 \pi} h_{K}(\dot{\tilde{z}}(t)) d t+\varepsilon h_{T}(\dot{\tilde{z}}(t)) d t \\
& =c(K)^{\frac{1}{2}}+\frac{\varepsilon}{2} \int_{0}^{2 \pi} h_{T}(\dot{\tilde{z}}(t)) d t .
\end{aligned}
$$

Rearranging (for fixed $\varepsilon>0$ and $p>1$ ), and applying equation (1.4.2), one gets that for any such $\tilde{z}$

$$
c(T)^{\frac{1}{2}} \leq \frac{c(K+\varepsilon T)^{\frac{1}{2}}-c(K)^{\frac{1}{2}}}{\varepsilon} \leq \frac{1}{2} \int_{0}^{2 \pi} h_{T}(\dot{\tilde{z}}(t)) d t .
$$


Note that the middle expression is a decreasing function of $\varepsilon$, which as $\varepsilon \rightarrow \infty$ converges to the left hand side, and as $\varepsilon \rightarrow 0^{+}$converges to $d_{T}(K) / 2 \sqrt{c(T)}$.

Next, note that $\tilde{z}$ is a critical point of the functional $I_{p_{1}}^{K}(z)=\int_{0}^{2 \pi} h_{K}^{p_{1}}(\dot{z}(t)) d t$ defined on the space $\mathcal{E}_{p_{1}}$ as well (see Corollary 2.3 above and the reasoning before it). Hence, we can use the transformation $\mathcal{F}$ defined in the proof of Proposition 2.2, to map $\tilde{z}$ to the corresponding capacity carrier $\tilde{l}$ of $K$. Moreover, from equalities (2.8) and (2.9) it follows that

$$
\dot{\tilde{l}}=c(K)^{\frac{1}{2}} J \dot{\tilde{z}}
$$

Thus, the above inequality takes the form

$$
c(T)^{\frac{1}{2}} \leq \frac{c(K+\varepsilon T)^{\frac{1}{2}}-c(K)^{\frac{1}{2}}}{\varepsilon} \leq \frac{1}{2} c(K)^{-\frac{1}{2}} \int_{0}^{2 \pi} h_{T}\left(J^{-1} \dot{\tilde{l}}(t)\right) d t .
$$

Since there is a one-to-one correspondence between critical points $\tilde{z}$ of the functional $I_{p_{1}}^{K}$ and closed characteristics $\tilde{l}$ on $\partial K$, we may in fact write the above inequality as

$$
c(T)^{\frac{1}{2}} \leq \frac{c(K+\varepsilon T)^{\frac{1}{2}}-c(K)^{\frac{1}{2}}}{\varepsilon} \leq \frac{1}{2} c(K)^{-\frac{1}{2}} \inf _{\tilde{l}} \int_{0}^{2 \pi} h_{T}\left(J^{-1} \dot{\tilde{l}}(t)\right) d t,
$$

where the infimum runs over all the loops $l$ which are images under $\mathcal{F}$ of $\tilde{z}$ minimizing equation (2.1) for $c(K)^{\frac{1}{2}}$ i.e., all the capacity carriers of $K$.

Thus, we arrive at

$$
\left.4 c(K) c(T) \leq \text { length }_{J T^{\circ}}(l)\right)^{2},
$$

for any capacity carrier $l$ on $\partial K$, proving Corollary [1.6, We may also take the limit in (4.2) as $\varepsilon \rightarrow 0^{+}$to see that

Corollary 4.2. For any $n$, any $K, T \in \mathcal{K}^{2 n}$, and any capacity carrier on $\partial K$, we have that

$$
d_{T}(K) \leq \inf _{\tilde{l}} \int_{0}^{2 \pi} h_{T}\left(J^{-1} \dot{\tilde{l}}(t)\right) d t=\operatorname{length}_{J T^{\circ}}(l) .
$$

Next we turn to the relation between the capacity and the Mean-Width of a body.

Proof of Corollary 1.7. We denote by $U(n)$ the group of unitary transformations in $\mathbb{C}^{n} \simeq \mathbb{R}^{2 n}$. Note that $c(U K)=c(K)$ for any unitary operator $U \in U(n)$. The Brunn-Minkowski inequality thus implies that for $U_{1}, U_{2} \in U(n)$

$$
c(K) \leq c\left(\frac{U_{1} K+U_{2} K}{2}\right)
$$

and by induction also

$$
c(K) \leq c\left(\frac{1}{N} \sum_{i=1}^{N} U_{i} K\right)
$$


Further, this is true also if we integrate (with respect to Minkowski addition) along the unitary group with respect to the uniform Haar measure $d \mu$ on this group:

$$
c(K) \leq c\left(\int U K d \mu(U)\right) .
$$

However, it is not hard to see that the integral on the left hand side is simply a Euclidean ball of some radius, since it is invariant under rotations $U \in U(n)$, and further, it is easy to determine its radius since $M^{*}(K)=M^{*}(U K)$, for $U \in U(n)$, and $M^{*}$ is an additive function with respect to Minkowski addition (for more details see [24]). Thus we have $\int U K d \mu(U)=M^{*}(K) B_{2}^{2 n}$, where $B_{2}^{2 n}$ is the Euclidean unit ball, and the inequality above translates to

$$
c(K) \leq c\left(M^{*}(K) B_{2}^{2 n}\right)=\pi\left(M^{*}(K)\right)^{2} .
$$

Next, we turn to the characterization of the equality case. Let $L$ denote the family of all capacity carriers on $\partial K$. Since we assume equality between the left and right hand side, we get equality throughout the following

$$
c(K) \leq \frac{1}{N^{2}} c\left(U_{1} K+\cdots+U_{N} K\right) \leq c\left(M^{*} B_{2}^{2 n}\right),
$$

for any $N$ and $U_{1}, \ldots, U_{N} \in U(n)$.

Applying the same argument as in the proof of Brunn-Minkowski for two summands (Theorem 1.4), this time to $N$ summands $K_{1}, \ldots K_{N}$ for arbitrary $N$, we get that the equality conditions becomes the following: there exists $N$ homothetic capacity carriers $l_{i} \subset \partial K_{i}$. Since in this case $K_{i}=U_{i} K$, we know that capacity carriers on $K_{i}$ are images by $U_{i}$ of capacity carriers on $K$. Moreover, since $K$ and $U_{i} K$ have the same capacity and are centrally symmetric, we see that if $l_{i}$ is a capacity carrier of $U_{i} K$ and is homothetic to a capacity carrier $l_{j}$ of $U_{j} K$, then they must actually be identical. We thus conclude that equality in Corollary 1.7 implies in fact that for every $N$ and $U_{1}, \ldots, U_{N} \in U(n)$ we have that

$$
U_{1} L \cap \cdots \cap U_{N} L \neq \emptyset \text {. }
$$

For any $K$ satisfying that $K \neq R B_{2}^{2 n}$ for any $R>0$ there exists some $\delta>0$ such that for every $\delta$-net $\mathcal{N}$ on $S^{2 n-1}$ we have that the restriction of $\|\cdot\|_{K}$ on $\mathcal{N}$ is not constant. Assume by contradiction that $K$ satisfies the equality in Corollary 1.7 but is not a Euclidean ball. Fix $\delta$ as above, and fix $C$ such that $C^{-1}|x| \leq\|x\|_{K} \leq C|x|$ for all $x$. Take $U_{1}, \ldots, U_{N}$ to be a $\delta$-net on $U(n)$ with respect to, say, the operator norm. The finiteness of $N$ follows from compactness on $U(n)$. Thus for every $U \in U(n)$ there is some $j$ such that $\left|U_{j} x-U x\right| \leq \delta|x|$ for all $x$.

It follows from (4.4) that there exists $l \in U_{1}^{-1} L \cap \cdots \cap U_{N}^{-1} L$. In particular, $U_{i} l \subset \partial K$, and so $\left\{U_{i} l(0)\right\}_{i=1}^{N} \subset \partial K$. Consider the set $\mathcal{N}=\left\{\frac{U_{i} l(0)}{\left|U_{i} l(0)\right|}\right\}_{i=1}^{N} \subset S^{2 n-1}$. 
Note that $\mathcal{N}$ is a $\delta$-net of $S^{2 n-1}$. However, on this set the norm is constant and equals $1 /|l(0)|$ ( since $U_{i}(l(0)) \in \partial K$ so $\left.\left\|U_{i}(l(0))\right\|_{K}=1\right)$. This is a contradiction to the choice of $\delta$, and we conclude that the norm $\|\cdot\|_{K}$ must have been Euclidean, completing the proof of Corollary 1.7 .

Proof of Corollary 1.8. Let $K, T \subset \mathcal{K}^{2 n}$ be general convex bodies, $x, y \in \mathbb{R}^{2 n}$ and $0 \leq \lambda \leq 1$. It is easy to verify that

$$
\lambda(K \cap(x+T))+(1-\lambda)(K \cap(y+T)) \subset(K \cap(\lambda x+(1-\lambda) y+T)) .
$$

Therefore, by monotonicity, we have that

$$
c^{1 / 2}(\lambda(K \cap(x+T))+(1-\lambda)(K \cap(y+T))) \leq c^{1 / 2}(K \cap(\lambda x+(1-\lambda) y+T)) .
$$

The Brunn-Minkowski inequality then implies that

$$
c^{1 / 2}(\lambda(K \cap(x+T)))+c^{1 / 2}((1-\lambda)(K \cap(y+T))) \leq c^{1 / 2}(K \cap(\lambda x+(1-\lambda) y+T)),
$$

and using homogeneity of capacity the proof of the general case is complete:

$$
\lambda c^{1 / 2}(K \cap(x+T))+(1-\lambda) c^{1 / 2}(K \cap(y+T)) \leq c^{1 / 2}(K \cap(\lambda x+(1-\lambda) y+T)) .
$$

For the symmetric case, let $y=-x$ and $\lambda=1 / 2$, we get

$$
(1 / 2) c^{1 / 2}(K \cap(x+T))+(1 / 2) c^{1 / 2}(K \cap(-x+T)) \leq c^{1 / 2}(K \cap T) .
$$

The second term on the left hand side equals to $c^{1 / 2}(-K \cap(x-T))$ (since $-I d$ is a symplectic map), which, by the symmetry assumptions on $K$ and $T$, is the same as $c^{1 / 2}(K \cap(x+T))$, the first term, and the inequality

$$
c^{1 / 2}(K \cap(x+T)) \leq c^{1 / 2}(K \cap T)
$$

is established,

\section{Proof of Lemma 2.5}

The proof is divided into three steps. We follow closely the arguments in [18] and [21].

First step: The functional $I$ is bounded from below on $\mathcal{E}$. Indeed, the function $h_{K}^{p}$ being continues and homogeneous of degree $p>1$ satisfies

$$
\frac{1}{\alpha}|y|^{p} \leq h_{K}^{p}(y) \leq \alpha|y|^{p}
$$

for some constant $\alpha \geq 1$, and thus

$$
I(z)=\int_{0}^{2 \pi} h_{K}^{p}(\dot{z}(t)) d t \geq \frac{1}{\alpha}\|\dot{z}\|_{p}^{p},
$$


where $\|\cdot\|_{p}$ stands for the $L_{p}$ norm on $S^{1}$. From Hölder's inequality it follows that for $z \in \mathcal{E}$

$$
2=\int_{0}^{2 \pi}\langle J z(t), \dot{z}(t)\rangle d t \leq\|z\|_{q}\|\dot{z}\|_{p}, \quad \text { where } \frac{1}{p}+\frac{1}{q}=1
$$

Using Poincaré inequality and the fact that $\int_{0}^{2 \pi} z(t) d t=0$, we deduce that

$$
\|z\|_{q} \leq\|z\|_{\infty} \leq 2 \pi\|\dot{z}\|_{1} \leq 2 \pi\|\dot{z}\|_{p}
$$

and hence $\frac{1}{\sqrt{\pi}} \leq\|\dot{z}\|_{p}$, which in turn implies that

$$
I(z)=\int_{0}^{2 \pi} h_{K}^{p}(\dot{z}(t)) d t \geq \frac{1}{\alpha}\|\dot{z}\|_{p}^{p} \geq \frac{\pi^{\frac{-p}{2}}}{\alpha}>0
$$

Second step: The functional $I$ attains its minimum on $\mathcal{E}$ namely, there exists $\tilde{z} \in \mathcal{E}$ with

$$
\int_{0}^{2 \pi} h_{K}^{p}(\dot{\tilde{z}}(t)) d t=\inf _{z \in \mathcal{E}} \int_{0}^{2 \pi} h_{K}^{p}(\dot{z}(t)) d t=\tilde{\lambda}>0
$$

To show this, we pick a minimizing sequence $z_{j} \in \mathcal{E}$ such that

$$
\lim _{j \rightarrow \infty} \int_{0}^{2 \pi} h_{K}^{p}\left(\dot{z}_{j}(t)\right) d t=\tilde{\lambda}
$$

It follows from (5.1), (5.2), and (5.3) that there exists some constant $C>0$ such that

$$
\frac{1}{C} \leq\left\|\dot{z}_{j}\right\|_{p} \leq C
$$

Moreover, from (5.2) it follows that

$$
\left\|z_{j}\right\|_{p} \leq\left\|\dot{z}_{j}\right\|_{p} \leq C
$$

In particular, $z_{j}$ is a bounded sequence in the Banach space $W^{1, p}\left(S^{1}, \mathbb{R}^{2 n}\right)$ and therefore, a subsequence, also denoted by $z_{j}$ converges weakly in $W^{1, p}\left(S^{1}, \mathbb{R}^{2 n}\right)$ to an element $z_{*} \in W^{1, p}\left(S^{1}, \mathbb{R}^{2 n}\right)$. Indeed, the closed unit ball of a reflexive Banach space is weakly compact and the space $W^{1, p}\left(S^{1}, \mathbb{R}^{2 n}\right)$, where $p>1$, is known to be reflexive (see e.g., [1]). We will show below that $z_{*} \in \mathcal{E}$. First we claim that $z_{j}$ converges uniformly to $z_{*}$ i.e.

$$
\sup _{t}\left|z_{j}(t)-z_{*}(t)\right| \rightarrow 0
$$

Indeed, the $z_{j}$ are uniformly continuous:

$$
\left|z_{j}(t)-z_{j}(s)\right| \leq\left|\int_{s}^{t} \dot{z}_{j}(\tau) d \tau\right| \leq|t-s|^{1 / q} C,
$$


and the claim follows from the Arzelà-Ascoli theorem. Next we claim that $z_{*} \in \mathcal{E}$. Indeed, even the weak convergence immediately implies that the mean value of $z_{*}$ vanishes. Moreover,

$$
2=\int_{0}^{2 \pi}\left\langle J z_{j}(t), \dot{z}_{j}(t)\right\rangle d t=\int_{0}^{2 \pi}\left\langle J\left(z_{j}(t)-z_{*}(t)\right), \dot{z}_{j}(t)\right\rangle d t+\int_{0}^{2 \pi}\left\langle J z_{*}(t), \dot{z}_{j}(t)\right\rangle d t
$$

The first term on the right hand side tends to zero by equation (5.5), Hölder inequality, and equation (5.4). The second term converges because of the weak convergence to

$$
\int_{0}^{2 \pi}\left\langle J z_{*}(t), \dot{z}_{*}(t)\right\rangle d t
$$

To see this, one must check that the linear functional $f(w)=\int_{0}^{2 \pi}\left\langle J z_{*}(t), \dot{w}(t)\right\rangle d t$ is bounded on $W^{1, p}\left(S^{1}, \mathbb{R}^{2 n}\right)$. This follows from Hölder's inequality as $z_{*} \in L_{q}\left(S^{1}, \mathbb{R}^{2 n}\right)$ (since $z_{*} \in W^{1, p}\left(S^{1}, \mathbb{R}^{2 n}\right)$ ). Thus the equation above, taking limit $j \rightarrow \infty$ takes the form

$$
\int_{0}^{2 \pi}\left\langle J z_{*}(t), \dot{z}_{*}(t)\right\rangle d t=2
$$

which implies that $z_{*} \in \mathcal{E}$. We now turn to show that $z_{*} \in \mathcal{E}$ is indeed the required minimum. We use the convexity of $h_{K}^{p}$ and deduce the point-wise estimate

$$
\left\langle\nabla h_{K}^{p}\left(\dot{z}_{j}(t)\right), \dot{z}_{*}(t)-\dot{z}_{j}(t)\right\rangle \leq h_{K}^{p}\left(\dot{z}_{*}(t)\right)-h_{K}^{p}\left(\dot{z}_{j}(t)\right) \leq\left\langle\nabla h_{K}^{p}\left(\dot{z}_{*}(t)\right), \dot{z}_{*}(t)-\dot{z}_{j}(t)\right\rangle,
$$

which gives

$$
\int_{0}^{2 \pi} h_{K}^{p}\left(\dot{z}_{*}(t)\right) d t-\int_{0}^{2 \pi} h_{K}^{p}\left(\dot{z}_{j}(t)\right) d t \leq \int_{0}^{2 \pi}\left\langle\nabla h_{K}^{p}\left(\dot{z}_{*}(t)\right), \dot{z}_{*}(t)-\dot{z}_{j}(t)\right\rangle d t
$$

To see that the right hand side of inequality (5.6) tends to zero, it is enough as before to check that $\nabla h_{K}^{p}\left(\dot{z}_{*}\right)$ belongs to $L_{q}\left(S^{1}, \mathbb{R}^{2 n}\right)$. Indeed, since $\nabla h_{K}^{p}$ is homogeneous of degree $p-1$ there exists some positive constant $K$ for which $\left|\nabla h_{K}^{p}(x)\right| \leq K|x|^{p-1}$, and hence it follows from equation (5.4) that

$$
\int_{0}^{2 \pi}\left|\nabla h_{K}^{p}\left(\dot{z}_{*}(t)\right)\right|^{q} d t=\int_{0}^{2 \pi}\left|\nabla h_{K}^{p}\left(\dot{z}_{*}(t)\right)\right|^{\frac{p}{p-1}} d t \leq K^{\frac{p}{p-1}} \int_{0}^{2 \pi}\left|\dot{z}_{*}(t)\right|^{p} d t<\infty .
$$

Thus the right hand side of inequality (5.6) tends to zero. Hence,

$$
\tilde{\lambda} \leq \int_{0}^{2 \pi} h_{K}^{p}\left(\dot{z}_{*}(t)\right) d t \leq \liminf _{j \rightarrow \infty} \int_{0}^{2 \pi} h_{K}^{p}\left(\dot{z}_{j}(t)\right) d t=\tilde{\lambda},
$$

and we have proved that $z_{*}$ is the minimum of $I(z)$ for $z \in \mathcal{E}$.

Third step: First we show that the critical points of $I$ satisfy the required Euler equation (2.4). Let $z$ be a critical point of $I$. Hence, according to Definition 2.4 for every $\xi \in W^{1, p}\left(S^{1}, \mathbb{R}^{2 n}\right)$ such that $\int_{0}^{2 \pi} \xi(t) d t=0, \int_{0}^{2 \pi}\langle J z(t), \dot{\xi}(t)\rangle d t=0$ we have that

$$
\int_{0}^{2 \pi}\left\langle\nabla h_{K}^{p}(\dot{z}(t)), \dot{\xi}(t)\right\rangle d t=0
$$


Next we choose a special $\xi$ namely such that $\dot{\xi}$ is of the form $\dot{\xi}=\nabla h_{K}^{p}(\dot{z})-\beta J z-\alpha$ where $\alpha$ is a vector and $\beta$ is a constant. The vector $\alpha$ is chosen so that $\xi(0)=\xi(2 \pi)$ namely

$$
\alpha=\frac{1}{2 \pi} \int_{0}^{2 \pi} \nabla h_{K}^{p}(\dot{z}(t)) d t
$$

In order to show that

$$
x(t)=\int_{0}^{t} \dot{\xi}(s) d s \in W^{1, p}\left(S^{1}, R^{2 n}\right),
$$

one uses a simple continuity properties of $\dot{\xi}$. We choose $\beta$ so that the condition

$$
\int_{0}^{2 \pi}\langle J z(t), \dot{\xi}(t)\rangle d t=0
$$

is satisfied. With this function $\xi$ we compute

$$
\int_{0}^{2 \pi}|\dot{\xi}(t)|^{2} d t=\int_{0}^{2 \pi}\left\langle\nabla h_{K}^{p}(\dot{z}(t)), \dot{\xi}(t)\right\rangle d t-\beta \int_{0}^{2 \pi}\langle J z(t), \dot{\xi}(t)\rangle d t-\left\langle\alpha, \int_{0}^{2 \pi} \dot{\xi}(t) d t\right\rangle=0
$$

Thus, the critical point $z$ satisfies the Euler equation $\nabla h_{K}^{p}(\dot{z})=\beta J z+\alpha$. Moreover, it follows from Euler formula that

$$
\lambda=\int_{0}^{2 \pi} h_{K}^{p}(\dot{z}(t)) d t=\frac{1}{p} \int_{0}^{2 \pi}\left\langle\nabla h_{K}^{p}(\dot{z}(t)), \dot{z}(t)\right\rangle d t=\frac{\beta}{p} \int_{0}^{2 \pi}\langle J z(t), \dot{z}(t)\rangle d t=\frac{2 \beta}{p},
$$

and hence $\beta=\frac{\lambda p}{2}$.

For the other direction, namely that any loop $z$ satisfying Euler equation (2.4) is a critical point of $I$, we simply check that for $\xi \in W^{1, p}\left(S^{1}, R^{2 n}\right)$ with $\int_{0}^{2 \pi} \xi(t) d t=0$ and $\int_{0}^{2 \pi}\langle J z(t), \dot{\xi}(t)\rangle d t=0$ we have

$$
\int_{0}^{2 \pi}\left\langle\nabla h_{K}^{p}(\dot{z}(t)), \dot{\xi}(t)\right\rangle d t=\int_{0}^{2 \pi}\left\langle\frac{\lambda p}{2} J z(t)+\alpha, \dot{\xi}(t)\right\rangle d t=0,
$$

as required. This concludes the proof of the Lemma.

\section{References}

[1] Adams, R.A. Sobolev Spaces, Pure and Applied Mathematics, Vol. 65. Academic Press, New York-London, 1975.

[2] Artstein-Avidan, S., Ostrover Y. On Symplectic Capacities and Volume Radius, Preprint math.SG/0603411

[3] Artstein-Avidan, S., Milman, V., Ostrover, Y. The M-ellipsoid, Symplectic Capacities and Volume, Preprint math.SG/0604434 
[4] Aubin, J.P., Ekeland, I. Applied nonlinear analysis. Pure and Applied Mathematics, New York, 1984

[5] Borell, C. Capacitary inequalities of the Brunn-Minkwoski type, Math. Ann. 263 (1983), 179-184.

[6] Brascamp, H.J., Lieb, E.H. On extensions of the Brunn-Minkowski and PrékopaLeindler theorem, including inequalities for log-concave functions, and with an application to the diffusion equation J. Funct. Anal., 22 (1976), 366-389.

[7] Cieliebak, K., Hofer, H., Latschev, J., Schlenk F. Quantitative symplectic geometry. math.SG/0506191.

[8] Clarke, F. A classical variational principle for periodic Hamiltonian trajectories, Proc. Amer. Math. Soc., 76:186-188, 1979.

[9] Clarke, F., Ekeland, I. Hamiltonian trajectories having prescribed minimal period, Comm. Pure Appl. Math. 33, 103-116 (1980)

[10] de Gosson, Maurice. Symplectic geometry and quantum mechanics, Operator Theory: Advances and Applications, 166. Advances in Partial Differential Equations (Basel). Birkhäuser Verlag, Basel, 2006.

[11] Ekeland, I. Convexity Methods in Hamiltonian Mechanics, Ergebnisse der Mathematik und ihrer Grenzgebiete (3), 19. Springer-Verlag, Berlin, 1990.

[12] Ekeland, I., Hofer, H. Symplectic topology and Hamiltonian dynamics, Math. Z. 200 (1989), no. 3, 355-378.

[13] Ekeland, I., Hofer, H. Symplectic topology and Hamiltonian dynamics II, Math. Z. 203 (1990), no.4, 553-567.

[14] Firey, Wm.J. p-Means of convex bodies, Math. Scand. 10 (1962), 17-24.

[15] Gardner, R.J. The Brunn-Minkowski inequality, Bull. Amer. Math. Soc. 39 (2002), 355-405.

[16] Gromov, M. Pseudoholomorphic curves in symplectic manifolds, Invent. Math. 82 (1985), no. 2, 307-347.

[17] Hofer, H. Symplectic capacities. Geometry of low-dimensional manifolds, 2 (Durham, 1989), 15-34, London Math. Soc. Lecture Note Ser., 151, Cambridge Univ. Press, Cambridge, 1990.

[18] Hofer, H., Zehnder, E. Symplectic Invariants and Hamiltonian Dynamics, Birkhäuser, Basel (1994). 
[19] Hofer, H., Zehnder, E. A new capacity for symplectic manifolds, Analysis et cetera. Academic press, 1990. Pages 405-428.

[20] Lalonde, F. Energy and capacities in symplectic topology. Geometric topology (Athens, GA, 1993), 328-374, AMS/IP Stud. Adv. Math., 2.1, Amer. Math. Soc., Providence, RI, 1997.

[21] Moser, J., Zehnder, E. Notes on Dynamical Systems, Courant Lecture Notes in Mathematics, 12. New York University (2005).

[22] McDuff, D. Symplectic topology and capacities, Prospects in mathematics (Princeton, NJ, 1996), 69-81, Amer. Math. Soc., Providence, RI, 1999.

[23] McDuff, D., Salamon, D. Introduction to Symplectic Topology, 2nd edition, Oxford University Press, Oxford, England (1998).

[24] Pisier, G. The volume of convex bodies and Banach space geometry, Cambridge Tracts in Mathematics, 94. Cambridge University Press, Cambridge, 1989.

[25] Rabinowitz, P. Periodic solutions of Hamiltonian systems, Comm. Pure Appl. Math., 31:157-184, 1978.

[26] Schneider, R. Convex bodies: the Brunn-Minkowski theory, Encyclopedia of Mathematics and its Applications, 44. Cambridge University Press, 1993.

[27] Siburg, K.F. Symplectic capacities in two dimensions, manusripta math., 78:149$163,1993$.

[28] Viterbo, C. Metric and isoperimetric problems in symplectic geometry. J. Amer. Math. Soc. 13 (2000), no. 2, 411-431.

[29] Viterbo, C. Capacités symplectiques et applications (d'après Ekeland-Hofer, Gromov). Séminaire Bourbaki, Vol. 1988/89. Astérisque no. 177-178 (1989), Exp. no. 714, 345-362.

[30] Weinstein, A. Periodic orbits for convex Hamiltonian systems, Ann. Math., 108:507-518, 1978.

Shiri Artstein-Avidan

School of Mathematical Sciences, Tel Aviv University, Tel Aviv 69978, Israel

e-mail: shiri@post.tau.ac.il

Yaron Ostrover

Department of Mathematics, M.I.T, Cambridge MA 02139, USA

e-mail: ostrover@math.mit.edu 